\title{
The Differential Method for Force Measurement Based on Electrostatic Force
}

\author{
Peiyuan Sun, ${ }^{1}$ Meirong Zhao, ${ }^{1}$ Jile Jiang, ${ }^{2}$ Yelong Zheng, ${ }^{1}$ Yaqian Han, ${ }^{1}$ and Le Song ${ }^{1,3}$ \\ ${ }^{1}$ State Key Laboratory of Precision Measuring Technology and Instruments, Tianjin University, Tianjin 300072, China \\ ${ }^{2}$ National Institute of Metrology, Beijing 100013, China \\ ${ }^{3}$ Center of MicroNano Manufacturing Technology, Tianjin University, Tianjin 300072, China \\ Correspondence should be addressed to Le Song; songle@tju.edu.cn
}

Received 8 December 2016; Revised 20 March 2017; Accepted 27 March 2017; Published 11 May 2017

Academic Editor: Dzung Dao

Copyright (c) 2017 Peiyuan Sun et al. This is an open access article distributed under the Creative Commons Attribution License, which permits unrestricted use, distribution, and reproduction in any medium, provided the original work is properly cited.

\begin{abstract}
The small force measurement is very important with development of the technology. The electrostatic force is adopted, in which a pair of coaxial cylindrical capacitors generate the electrostatic force when a voltage is applied across the inner and outer electrodes. However, the measured force will be covered by noise (creep, ground vibration, and air flow) and could not be measured accurately. In this paper, we introduce the differential method to reduce the effect of noise. Two identical parallelogram mechanisms (PM) serve as the mechanical spring. One of the PM serves as the reference and another serves as the force sensor. The common signal will be offset, and the difference signal will serve as output. In this way, the effect of the creep will be reduced. The measurement system of the electrostatic force was characterized by applying mechanical forces of known magnitude via loading weights of calibrated masses. The uncertainty from voltage, laser interferometer, and capacitance gradient was estimated. For the measured force, the relative uncertainty is less than $4 \%\left(k_{p}=2\right)$.
\end{abstract}

\section{Introduction}

The small force measurement is very important with development of the technology [1-19]. It involves precision instrument, MEMS [1, 2], biology [12, 13], magnetic field distributions $[14,15]$, and tribological properties of material [1619]. Scientific research institutes have carried out researches from country to country. The National Institute of Standards and Technology (NIST) in USA created a force measurement system based on the electrostatic force, having a resolution of $15 \mathrm{nN}[3,4]$. The sensitivity of the system was constrained by noise. To reduce the vibration, the system of NIST was built at $12 \mathrm{~m}$ of the underground to reduce the vibration. The Physikalisch Technische Bundesanstalt (PTB) in Germany nano-Newton force metrology group designed an aluminum plate pendulum; the resolution of the setup was constrained by noise. PTB set up two identical systems to reduce the noise. This setup could measure forces less than $10-5 \mathrm{~N}$, whose resolution is $10-12 \mathrm{~N}[5,6]$. The National Physical Laboratory (NPL) developed a force balance with working range from $10^{-9} \mathrm{~N}$ to $10^{-6} \mathrm{~N}[7,8]$. The Korean Research Institute of
Standards and Science (KRISS) built a nanoforce calibrator that realized the calibration of cantilevers whose stiffness ranges within $0.01 \sim 100 \mathrm{~N} / \mathrm{m}$, with a relative standard uncertainty of $1 \%[9,10]$.

The noise (creep, ground vibration, and air flow) is one of limits for force resolution. The measured force will be covered by noise and could not be measured accurately. Improving the environment is one way to reduce the effect of noise. Song introduced air damping to improve environmental noise suppression [11]. In this paper, we introduce the differential method force measurement (DFM) to reduce the effect of noise. With comparison with another method, DFM is more convenient and has lower demands for applying environment. What is more, DFM could be applied combined with other methods above to reduce noise further.

\section{Results}

2.1. The Electrostatic Force System. The electrostatic force is adopted, in which a pair of coaxial cylindrical capacitors 


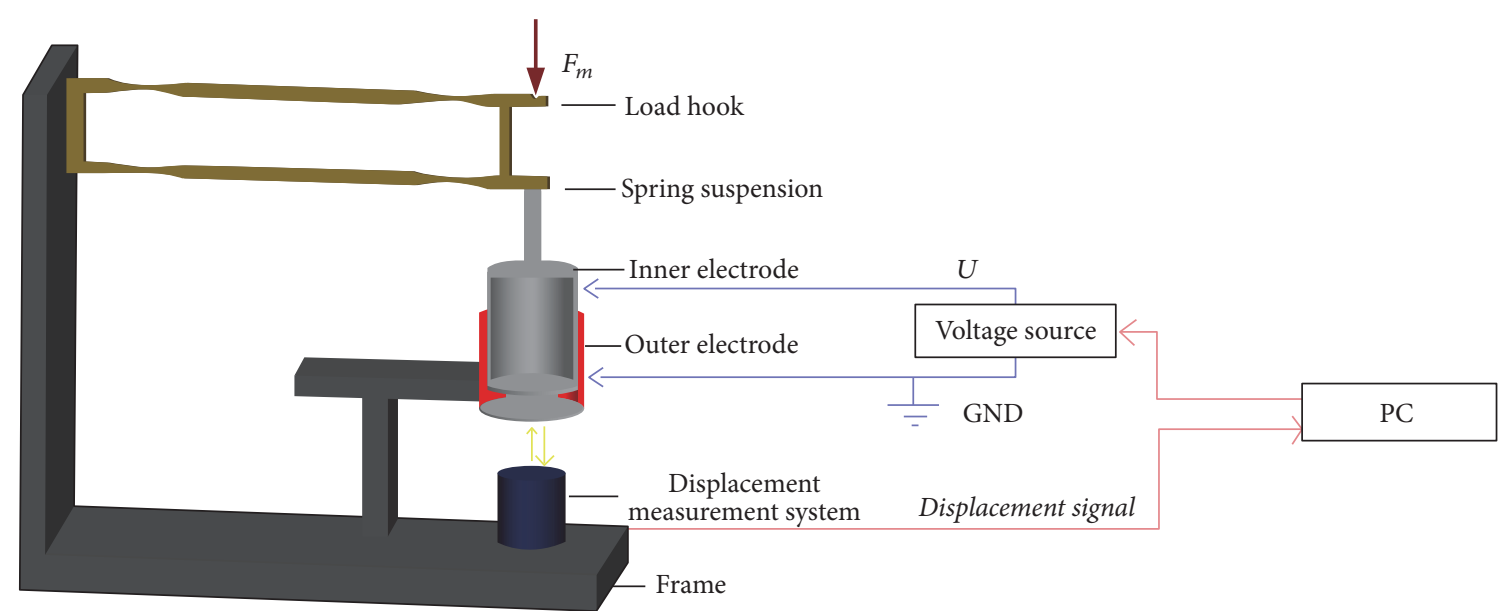

FIGURE 1: The schematic diagram of force measurement system based on electrostatic force.

generate the electrostatic force when a voltage is applied across the inner and outer electrodes [20-22]. The relationship between the applied voltage and the electrostatic force is given by

$$
F_{e}=\frac{d C}{2 d z}\left(U+U_{s}\right)^{2}
$$

where $U$ is the voltage applied across the inner and outer electrodes; $F_{e}$ is the generated electrostatic force; $U_{s}$ is the potential difference between the electrodes resulting from the hypothesized surface field effects; $d C / d z$ is the capacitance gradient:

$$
\frac{d c}{d z}=\frac{2 \pi \varepsilon}{\ln R / r}
$$

where $R$ represents the radius of the inner electrode, $r$ represents the radius of the outer electrode, and $\varepsilon$ is the absolute permittivity.

The inner electrode, which is fixed at the end of parallelogram mechanism, moves freely along the vertical direction and there is not a constraint of the displacement, whereas the outer electrode is fixed, as shown in Figure 1.

To improve the sensitivity of the force measurement, the stiffness of the spring should be as smaller as possible. While the small stiffness will lead to the creep, the output of the displacement is about $1 \mu \mathrm{m} / \mathrm{h}$. The weight of inner electrode and its connection is about $20 \mathrm{~g}$; the stress also will result in creep. In another way, the ground vibration and air flow will affect the measurement results.

2.2. The Differential Method. To reduce the effect of the noise, a differential system is built as shown in Figure 2. Two identical parallelogram mechanisms (PM) serve as the mechanical spring [23-25] as shown in Figure 2(a). Four notch pivots, which are circle notch, are needed and carved into the stage in order to achieve rectilinear motion capability as shown in Figure 2(b). At its thinnest point the thickness is $t=0.2 \mathrm{~mm}$; it has a width of $b=5 \mathrm{~mm}$, a cutting radius of $R=5 \mathrm{~mm}$, and $l=$ $70 \mathrm{~mm}$. The stiffness of the flexible hinge, $K$, is measured as $10 \mathrm{~N} / \mathrm{m}$. One of the PM serves as the reference; another serves as the force sensor. The two-displacement signal was input into RLE20. The common signal will be offset, and the difference signal will serve as PID input. In this way, the effect of the creep will be reduced.

The creep of the PM, $\delta$, increased with the load; the relationship between $\delta$ and time $t$ is shown as in

$$
\delta=\frac{\sigma}{k}\left(1-e^{-(k / \xi) t}\right),
$$

where $\sigma$ is stress of PM and $k$ and $\xi$ are stiffness and damping. The stress of $\mathrm{PM}$ is about $0.4 \mathrm{MPa}$, for the mass of the inner electrode, connection is $20 \mathrm{~g}$, and the thinnest of the PM is $5 \times 0.1 \mathrm{~mm}^{2}$. If the parameters of two PMs are identical, which is very difficult in machining, the creep could be completely offset. But the stress will be changed with different measurement force. So the creep will alter with time.

The device was shown in Figure 3(a); to eliminate the ground noise, the device is on the isolated platform. To test the performance of the differential system, the standard weights (1 mg, $10 \mathrm{mg}$, and $100 \mathrm{mg}$ ) were loaded on the system. The creep under different loads is sampled by the displacement of PM which is measured by lase interferometer with $20 \mathrm{~Hz}$. The result of 50 minutes is shown in Figure 3(b). The creep of the nondifferential system is very large, about $1 \mu \mathrm{m} / \mathrm{h}$, while in differential system, the creep is $0.05 \mu \mathrm{m} / \mathrm{h}, 0.1 \mu \mathrm{m} / \mathrm{h}$, and $0.2 \mu \mathrm{m} / \mathrm{h}$ at load of $1 \mathrm{mg}, 10 \mathrm{mg}$, and $100 \mathrm{mg}$, respectively. The creep of differential system is much smaller than nondifferential system. To test the dynamic performance, the PM is put in a free vibration state by a pulse excitation. The vibration of the inner electrode is recorded using a laser interferometer with a sampling frequency of $100 \mathrm{~Hz}$. The sampling lasts for $20 \mathrm{~s}$ as shown in Figure 3(c). The original amplitude for the system is about $0.25 \mu \mathrm{m}$. The system takes $10 \mathrm{~s}$ before it reaches stable state.

2.3. The Capacitance Gradient Test. The capacitance gradient test is implemented before force measurement. According to (2), the electrostatic force is linear to $d C / d z$ with certain voltage. The inner electrode stays motionless while the outer 


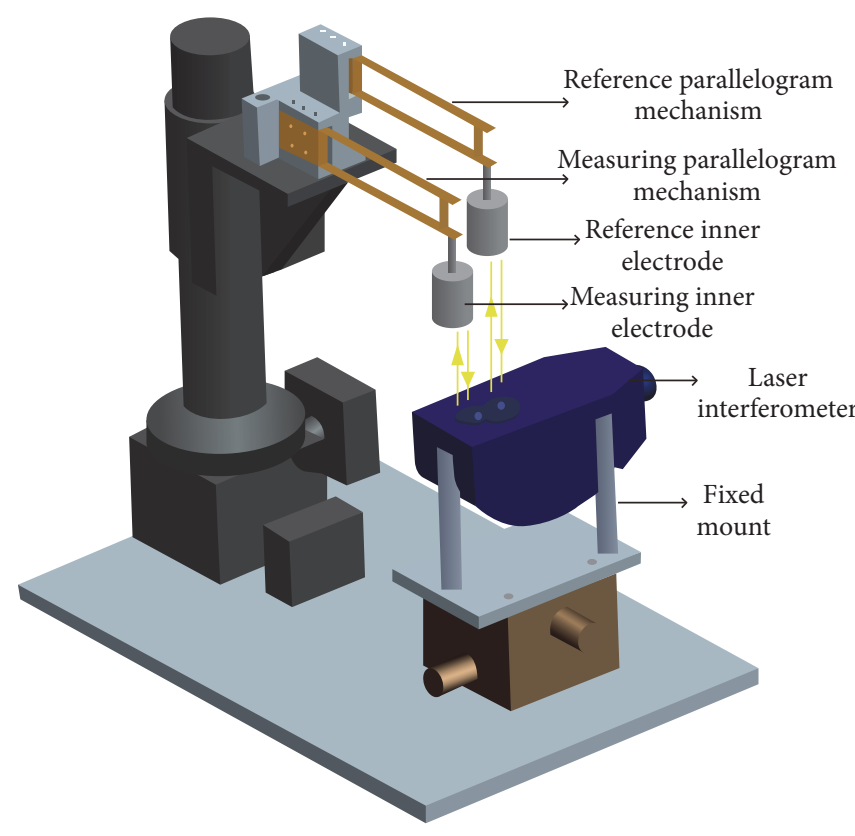

(a)

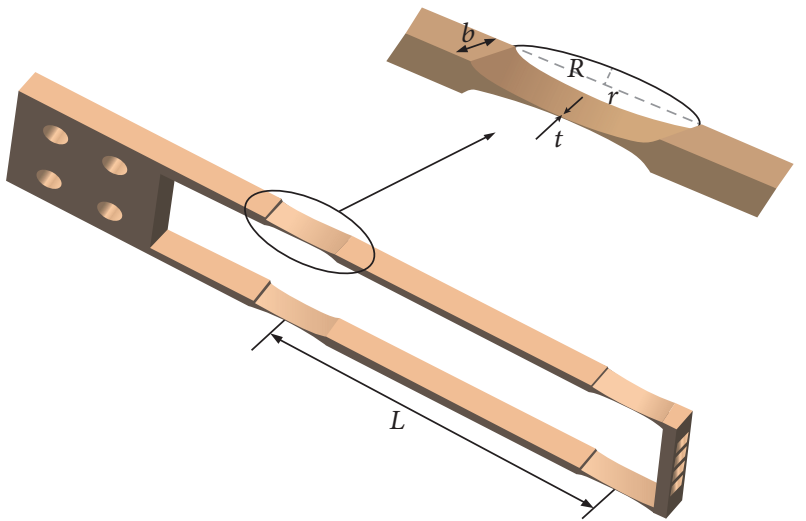

(b)

FIgURE 2: The differential method to reduce the creep. (a) The schematic of differential method. (b) Identical parallelogram mechanisms with four notch pivots.

electrode elevates along with a lifting stage to measure $d C / d z$. The step of the outer electrode is $20 \mu \mathrm{m}$ and lasts $30 \mathrm{~s}$ for each step. A cycle includes 6 steps, as shown in Figure 4(a).

$C$ is captured by capacitance bridge AH2700 (with resolution $1 \mathrm{aF}$ ) and the displacement $z$ is measured by lase interferometer. The relative capacitance is shown in Figure 4(b). The capacitance-displacement data were fitted using a least squares straight line, and $d C / d z$ can be determined from the gradient of the line, as shown in Figure 4(c). From the experimental results, the average of $d C / d z$ was calculated as $0.9164 \mathrm{pF} / \mathrm{mm}$, with relative standard deviation being $0.03 \%$. The uncertainty of $d C / d z$ is listed in Table 1 and will be discussed in detail in Section 3.

2.4. The Comparison between the Electrostatic Force and Weight of Mass. Null balance was adopted to measure loaded force. These two forces are balanced by changing the voltage with the constant $d C / d z$. And a discretized proportionalintegral-derivative (PID) controller was used to achieve the task of controlling the position of the inner electrode [26, 27]. The block diagram of controlling system is shown as in Figure 1.

To evaluate the electrostatic force applied to achieve this equilibrium, the voltage signal is averaged over a period of $10 \mathrm{~s}$, starting from the time when the displacement is fully compensated. The resolution of the system was tested by measuring the voltage output without loading any force. In this way, the noise from the environment (ground vibration, thermal noise, and air flow) and thermal of electronics were accounted for. Repeating the described procedure twenty times, the standard deviation was $9 \mathrm{nN}$, as shown in Figure 5(a).
TABLE 1: Comparison of electrostatic force and mass.

\begin{tabular}{lccccc}
\hline $\begin{array}{l}\mathrm{G} \\
(\mu \mathrm{N})\end{array}$ & $\begin{array}{c}F_{e} \\
(\mu \mathrm{N})\end{array}$ & $\begin{array}{c}\text { Variance } \\
(\mu \mathrm{N})\end{array}$ & $\begin{array}{c}\text { Relative } \\
\text { variance } \\
(\%)\end{array}$ & $\begin{array}{c}\mathrm{G}-F_{e} \\
(\mu \mathrm{N})\end{array}$ & $\begin{array}{c}\text { Relative } \\
\text { deviation } \\
(\%)\end{array}$ \\
\hline 24.49 & 23.8 & 0.08 & 0.4 & 0.69 & 2.8 \\
32.06 & 33.1 & 0.3 & 0.9 & -1.04 & -3.2 \\
55.24 & 55.7 & 0.5 & 0.9 & -0.46 & -0.8 \\
75.62 & 76.7 & 0.6 & 0.8 & -1.08 & -1.4 \\
\hline
\end{tabular}

To evaluate the accuracy and the repeatability of the tested system, the relative deviation was evaluated. The gravity of a $1 \mathrm{mg}$ mass is $9.801 \mu \mathrm{N}$. The system has a loading button with a $\mathrm{V}$-groove for holding the wire weights. Repeating the procedure 20 times, the results for a $1 \mathrm{mg}$ standard weight had a mean value of $9.84 \mu \mathrm{N}$ with a standard deviation of $0.05 \mu \mathrm{N}$.

The measurement system of the electrostatic force was characterized by applying mechanical forces of known magnitude via loading weights of calibrated masses $(24.49,32.06$, 55.24 , and $75.62 \mu \mathrm{N})$. Here, $g=9.801 \mathrm{~N} / \mathrm{kg}$. The mass artifact employed in this prototype is stainless. The result of artifact $(24.49 \mu \mathrm{N})$ is shown in Figure 5(b), and detailed results are shown in Table 1.

\section{Discussion}

There are many factors that will affect the measurement results, such as the environment (ground vibration, thermal noise, and air flow), $d C / d z$, voltage, and laser interferometer. 

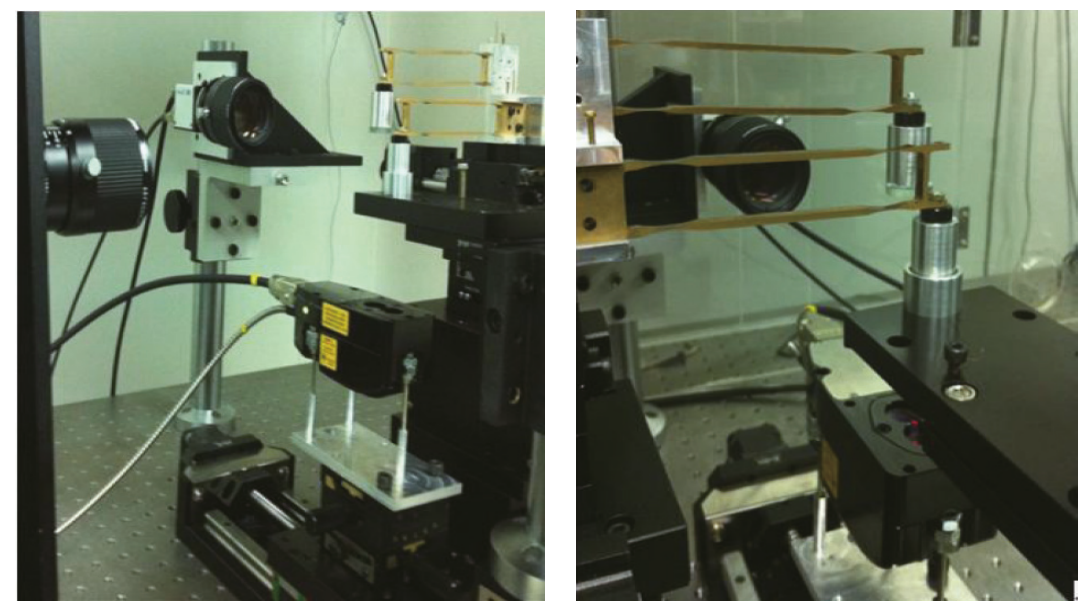

(a)

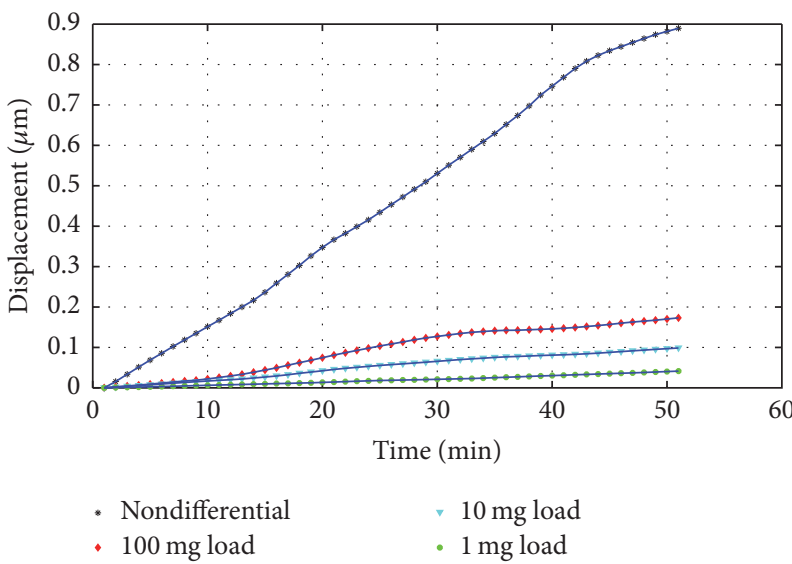

(b)

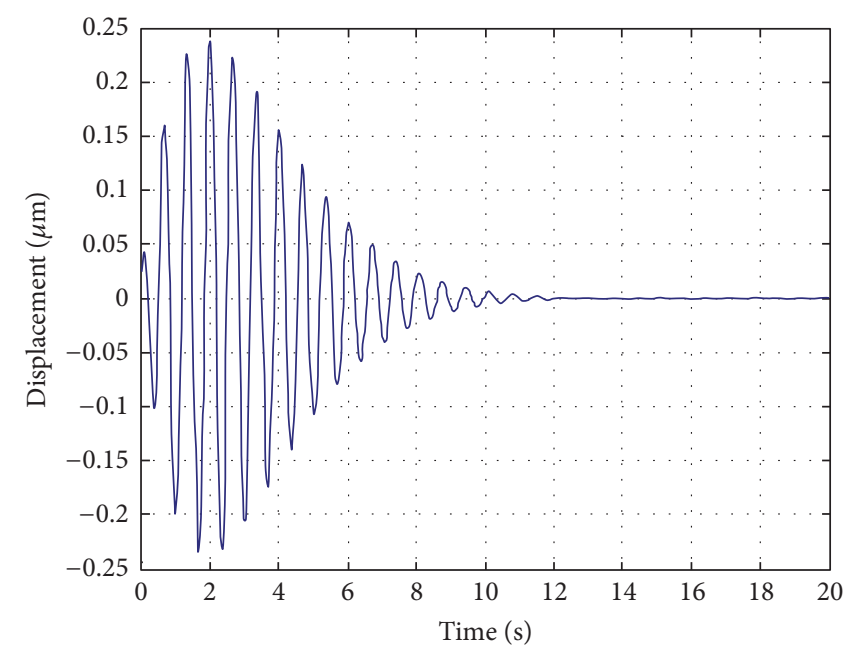

(c)

FIgURE 3: The device of differential system and performance test. (a) The device of differential system. (b) The creep of the differential system with difference load on the system. (c) The dynamic performance of the differential system.

Type A uncertainties of force are taken as the set standard deviation for a data run. The error of force, $\Delta f$, is shown as follows:

$$
\begin{aligned}
& \Delta f=\frac{\partial f}{\partial(d C / d z)} \Delta\left(\frac{d C}{d z}\right)+\frac{\partial f}{\partial U} \Delta u U, \\
& \Delta f=0.5 U^{2} \Delta\left(\frac{d C}{d z}\right)+U \frac{d C}{d z} \Delta U .
\end{aligned}
$$

Type B uncertainties of force are calculated as follows:

$$
u(f)=\sqrt{\left[0.5 U^{2}\left(\frac{d C}{d z}\right)\right]^{2}+\left[U \frac{d C}{d z} u(U)\right]^{2}} .
$$

The uncertainty of $d C / d z, u(d C / d z)$, comes from the electrode surface, the posture of the two electrodes, and the measurement of the gradient. The roughness of the surface would lead to the nonuniform distribution of the charge and come into uncertainty of $d C / d z$. In another way, for the $d C / d z$ is dependent on the gap of electrodes, the roughness also has an effect on $d C / d z$. The dimensional tolerances of $R$ and $r$ are confined to $3 \mu \mathrm{m}$, while both cylindrical form deviations were below $1 \mu \mathrm{m}$. So, the uniformity of the energy density of electric field between electrodes was ensured. The position of the electrodes was measured by CCD $(2448 \times$ 2050 pixels). To make the edge of the electrodes clear and easy to obtain, an LED optical source is placed opposite the camera for the exposure. By this setup, deviations regarding eccentricity and tilting of the electrode can be detected to values of $b=3 \mu \mathrm{m}$ and $\delta_{\text {sf }}=0.3^{\circ}$. Therefore, the maximum relative deviations of the capacitance gradient caused by eccentricity and tilt error are $0.0018 \%$ and $0.05 \%$. The relative standard deviation of $d C / d z$ is $0.03 \%$. Capacitance bridge and laser interferometer could be neglected. The uncertainty coming from $d C / d z$ is $0.06 \%$ as shown in Table 2 . 


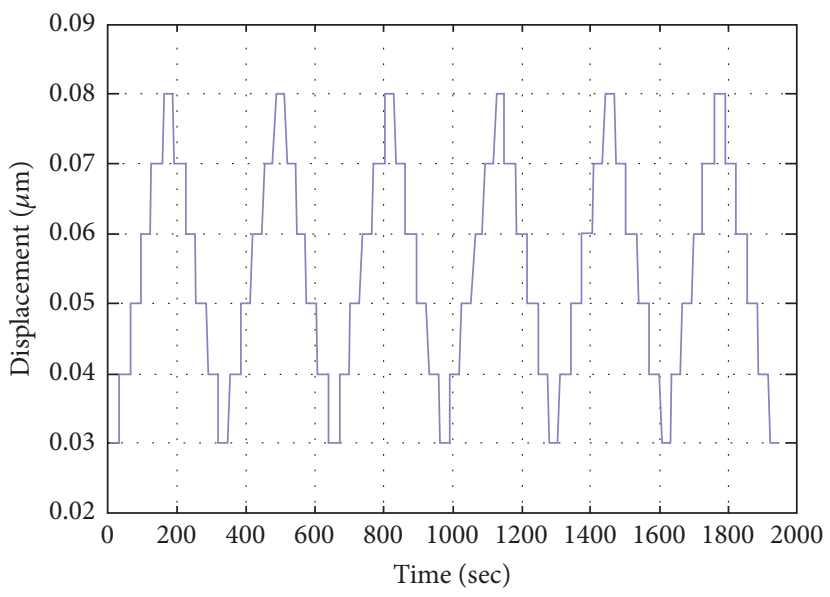

(a)

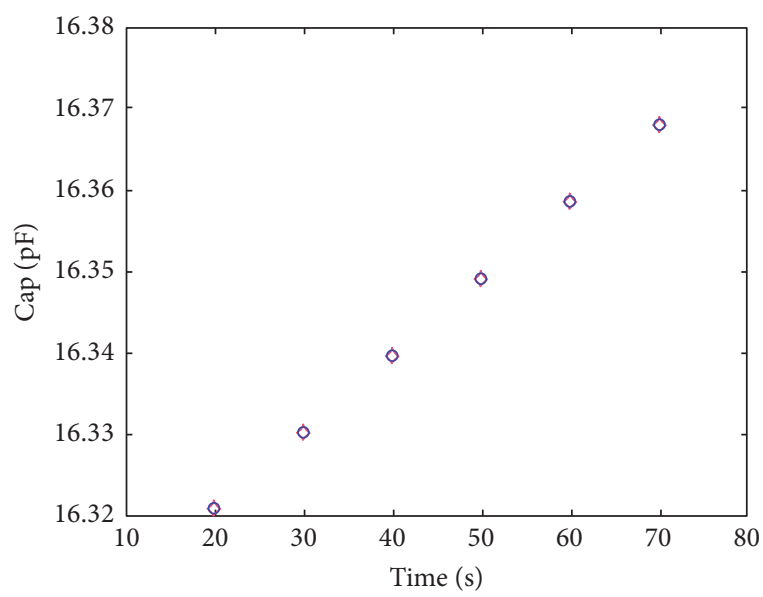

(b)

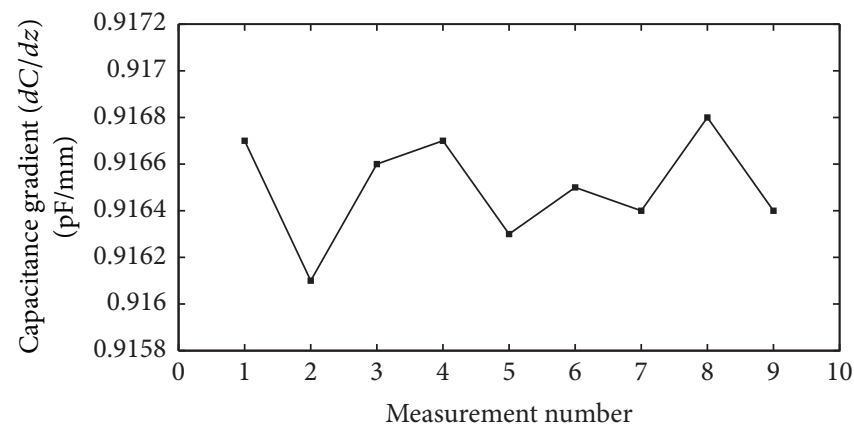

(c)

Figure 4: The capacitance gradient measurement result. (a) The displacement output of 6 cycles includes 6 steps. (b) Capacitancedisplacement curves of 6 cycles. (c) $d C / d z$ results fitted by a least squares straight line.

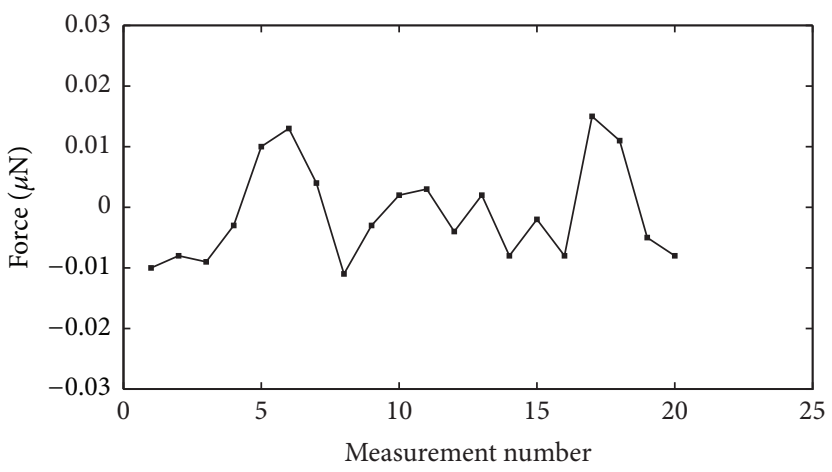

(a)

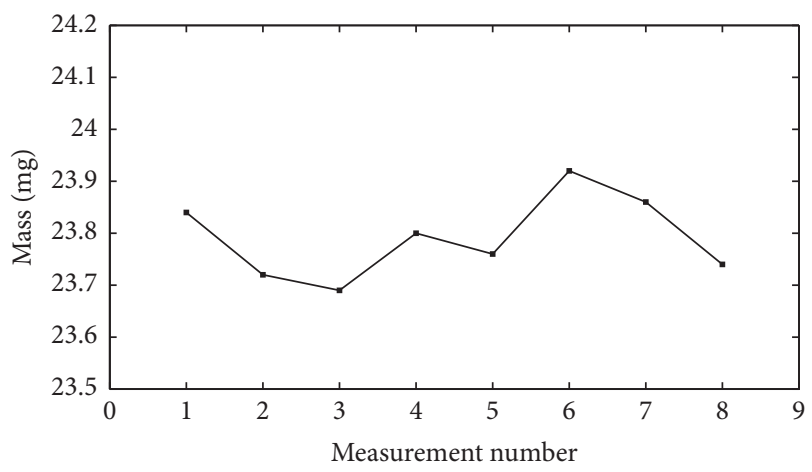

(b)

Figure 5: (a) The resolution test of the system. (b) The comparison of the electrostatic force and weights of calibrated masses (24.49 mg).

$U$ was supplied by Keithley 2410c voltage source with a resolution of $0.01 \mathrm{~V}$ and a maximum permissible error of $0.021 \mathrm{~V}$. The uncertainty coming from $U$ is $\sqrt{2 f(d C / d z)} d(U) / f$. Submitting $d C / d z=0.91 \mathrm{pF} / \mathrm{mm}$ and $d(U)$, the relative uncertainty is $\sqrt{4.1 / 406 f^{3}} \%$.

The uncertainty coming from the laser interferometer is estimated by its resolution $(1 \mathrm{~nm})$, the $3 \sigma$ method is adopted, and the error of laser interferometer is $d(d)=3 \mathrm{~nm}$. For the null balance is used in the measurement system, if the feedback signal has deviation to the real initial position, the inner electrode would not be restored to the initial position. The uncertainty coming from this factor could be estimated by $u(d)=K * d(d)$. In this system, $K=10 \mathrm{~N} / \mathrm{m}$, $u(d)=0.03 \mu \mathrm{N}$. The relative uncertainty coming from laser interferometer is $(3 / f) \%$ for the measured force $f(\mu N)$.

The standard uncertainties and corresponding data are listed in Table 3. The stated combined total uncertainty is determined by taking the root sum square of the contributions of individual uncertainty sources to the total uncertainty. 
TABLE 2: Uncertainty of capacitance gradient.

\begin{tabular}{lc}
\hline Uncertainty sources & Contribution to total uncertainty (\%) \\
\hline Electrode alignment & 0.05 \\
The repeatability & 0.03 \\
Capacitance bridge & Negligible \\
Displacement & Negligible \\
Combined & 0.06 \\
\hline
\end{tabular}

TABLE 3: Total uncertainty of force.

\begin{tabular}{lc}
\hline Uncertainty sources & Contribution (\%) \\
\hline Voltage & $\sqrt{4.1 / 406 f^{3}}$ \\
Capacitance gradient & 0.06 \\
Lase interferometer & $3 / f$ \\
Repeatable & 0.9 \\
Combined & 2 \\
\hline
\end{tabular}

According to Table 3, for the measured force $f=10 \mu \mathrm{N}$, the relative uncertainty is less than $2 \%$. The extended uncertainty of force is $4 \%\left(k_{p}=2\right)$. The uncertainty analysis agreed with the measurement results in Table 1.

\section{Conclusion}

We have described a differential method to reduce the effect of noise. Two identical PMs serve as the mechanical spring. The creep of the differential system was much better than that of nondifferential system. The creep of system decreased from $1 \mu \mathrm{m} / \mathrm{h}$ to $0.05 \mu \mathrm{m} / \mathrm{h}$ by adopting nondifferential method. The performance of the system was demonstrated through applying mechanical forces of known magnitude via loading weights of calibrated masses. The relative force measurement is less than $4 \%\left(k_{p}=2\right)$ according to the analysis experimental or theoretical. These results could help understanding the mechanism of creep and guiding the design of small force measurement system.

\section{Conflicts of Interest}

The authors declare that they have no conflicts of interest.

\section{Acknowledgments}

This study was supported by the Tianjin Natural Science Foundation (no. 17JCYBJC19000) and National Key Technology Research and Development Program of the Ministry of Science and Technology of China (no. 2011BAK15B06).

\section{References}

[1] M. Tamazin, A. Noureldin, and M. J. Korenberg, "Robust modeling of low-cost MEMS sensor errors in mobile devices using fast orthogonal search," Journal of Sensors, vol. 2013, Article ID 101820, 8 pages, 2013.

[2] J. X. Ren, R. Zhang, and B. Xu, "Adaptive fuzzy sliding mode control of MEMS gyroscope with finite time convergence," Journal of Sensors, vol. 2016, Article ID 1572303, 7 pages, 2016.
[3] J. R. Pratt, J. A. Kramar, D. B. Newell, and D. T. Smith, "Review of SI traceable force metrology for instrumented indentation and atomic force microscopy," Measurement Science and Technology, vol. 16, no. 11, pp. 2129-2137, 2005.

[4] R. Seugling, J. R. Pratt, D. B. Newell, and J. A. Kramar, "Realizing and disseminating the SI micronewton with the next generation NIST electrostatic force balance," in Proceedings of the ASPE Annual Meeting, Orlando, Fla, USA, October 2004.

[5] V. Nesterov, S. Buetefisch, and L. Koenders, "A nanonewton force facility to test Newton's law of gravity at micro- and submicrometer distances," Annalen der Physik, vol. 525, no. 89, pp. 728-737, 2013.

[6] V. Nesterov, "Facility and methods for the measurement of micro and nano forces in the range below $10-5 \mathrm{~N}$ with a resolution of 10-12 N (development concept)," Measurement Science and Technology, vol. 18, no. 2, article S06, pp. 360-366, 2007.

[7] R. Leach, D. Chetwynd, L. Blunt et al., "Recent advances in traceable nanoscale dimension and force metrology in the UK," Measurement Science and Technology, vol. 17, no. 3, pp. 467-476, 2006.

[8] R. K. Leach, J. Claverley, C. Giusca et al., "Advances in engineering nanometrology at the National Physical Laboratory," Measurement Science and Technology, vol. 23, no. 7, Article ID 074002, 2012.

[9] M.-S. Kim, J. R. Pratt, U. Brand, and C. W. Jones, "Report on the first international comparison of small force facilities: a pilot study at the micronewton level," Metrologia, vol. 49, no. 1, pp. 70-81, 2012.

[10] M.-S. Kim and J. R. Pratt, "SI traceability: current status and future trends for forces below 10 microNewtons," Measurement: Journal of the International Measurement Confederation, vol. 43, no. 2, pp. 169-182, 2010.

[11] Y. Zheng, L. Song, G. Hu et al., "Improving environmental noise suppression for micronewton force sensing based on electrostatic by injecting air damping," The Review of Scientific Instruments, vol. 85, no. 5, Article ID 055002, 7 pages, 2014.

[12] L. Wang and Q. Zhou, "Nepenthes pitchers: surface structure, physical property, anti-attachment function and potential application in mechanical controlling plague locust," Chinese Science Bulletin, vol. 59, no. 21, pp. 2513-2523, 2014.

[13] L. Wang and Q. Zhou, "Friction Force of locust Locusta migratoria manilensis (orthoptera, locustidae) on slippery zones of pitchers from four nepenthes species," Tribology Letters, vol. 44, no. 3, pp. 345-353, 2011.

[14] H. Xinjing, C. Shili, G. Shixu, Z. Wei, and J. Shijiu, "Magnetic charge and magnetic field distributions in ferromagnetic pipe," Applied Computational Electromagnetics Society Journal, vol. 28, no. 8, pp. 737-746, 2013.

[15] H. Xinjing, C. Shili, G. Shixu, Z. Wei, and J. Shijiu, "Analyses and application of the magnetic field at girth welds in pipelines," Measurement Science \& Technology, vol. 24, no. 11, p. 10, 2013.

[16] C. L. Dong, C. Q. Yuan, X. Q. Bai, X. P. Yan, and Z. Peng, "Tribological properties of aged nitrile butadiene rubber under dry sliding conditions," Wear, vol. 322-323, pp. 226-237, 2015.

[17] C. Dong, C. Yuan, X. Bai, X. Yan, and Z. Peng, "Study on wear behaviour and wear model of nitrile butadiene rubber under water lubricated conditions," RSC Advances, vol. 4, no. 36, pp. 19034-19042, 2014.

[18] L. Gao, Y. Liu, T. Ma, R. Shi, Y. Hu, and J. Luo, "Effects of interfacial alignments on the stability of graphene on $\mathrm{Ru}(0001)$ substrate," Applied Physics Letters, vol. 108, no. 26, Article ID 261601, 2016. 
[19] L. Gao, X. Ding, T. Lookman, J. Sun, and E. K. H. Salje, "Metastable phase transformation and hcp- $\omega$ transformation pathways in $\mathrm{Ti}$ and $\mathrm{Zr}$ under high hydrostatic pressures," Applied Physics Letters, vol. 109, no. 3, Article ID 031912, 2016.

[20] Y. L. Zheng, L. Song, G. Hu et al., "The multi-position calibration of the stiffness for atomic-force microscope cantilevers based on vibration," Measurement Science \& Technology, vol. 26, no. 5, p. 055001, 2015.

[21] L. Song, Y. Zheng, G. Hu et al., "Highly sensitive, precise, and traceable measurement of force," Instrumentation Science and Technology, vol. 44, no. 4, pp. 386-400, 2016.

[22] Y. Zheng, X. Yang, M. Zhao, T. Guan, and M. Jiang, "Adaptive control Algorithm improving the stability of micro force measurement system," Lecture Notes in Electrical Engineering, vol. 246, pp. 1183-1191, 2014.

[23] M. I. Mohamed, E. H. Hasan, and G. Aggag, "Study of creep behavior of load cells," Measurement: Journal of the International Measurement Confederation, vol. 42, no. 7, pp. 1006-1010, 2009.

[24] N. Lobontiu, J. S. N. Paine, E. Garcia, and M. Goldfarb, "Cornerfilleted flexure hinges," Journal of Mechanical Design, vol. 123, no. 3, pp. 346-352, 2001.

[25] V. Nesterov and U. Brand, "Modelling and investigation of the mechanical and electrical characteristics of the silicon 3D-boss microprobe for force and deflection measurements," Journal of Micromechanics and Microengineering, vol. 16, no. 7, article 003, pp. 1116-1127, 2006.

[26] S. Chen and S. Pan, "A force measurement system based on an electrostatic sensing and actuating technique for calibrating force in a micronewton range with a resolution of nanonewton scale," Measurement Science and Technology, vol. 22, no. 4, pp. 45104-45111, 2011.

[27] S.-J. Chen and S.-S. Pan, "Nanonewton force generation and detection based on a sensitive torsion pendulum," IEEE Transactions on Instrumentation and Measurement, vol. 58, no. 4, pp. 897-901, 2009. 


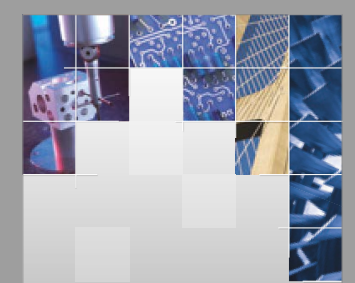

\section{Enfincering}
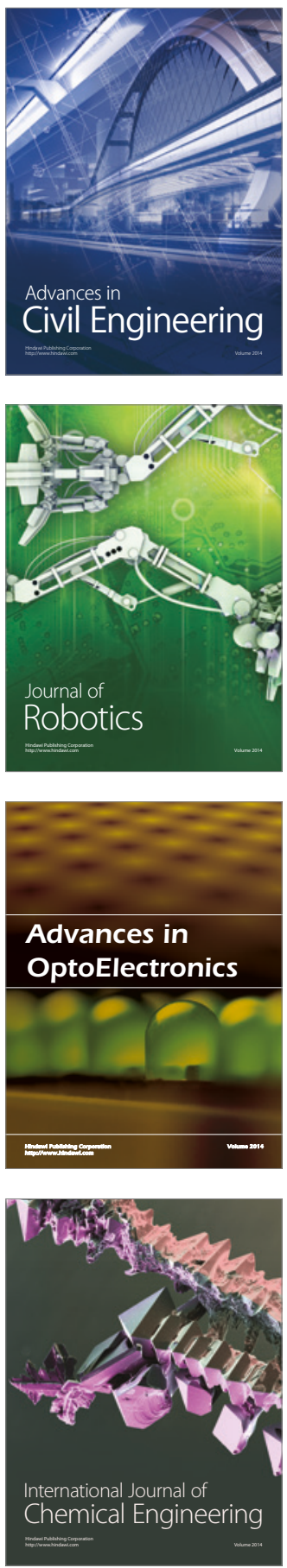

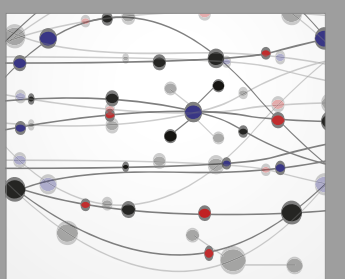

The Scientific World Journal

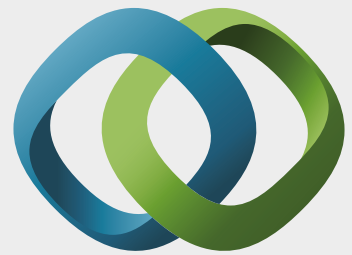

\section{Hindawi}

Submit your manuscripts at

https://www.hindawi.com
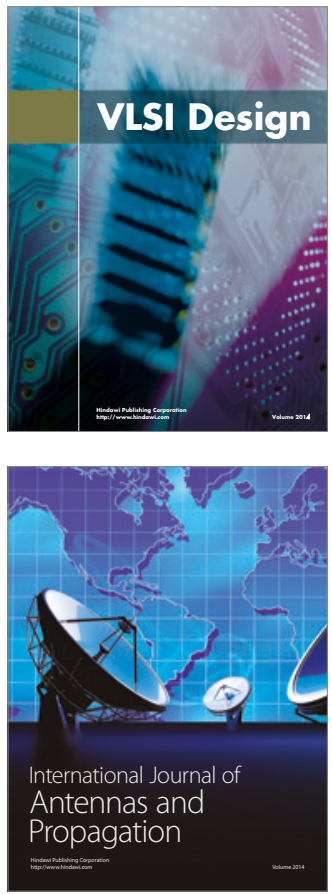

\section{Rotating}

Machinery
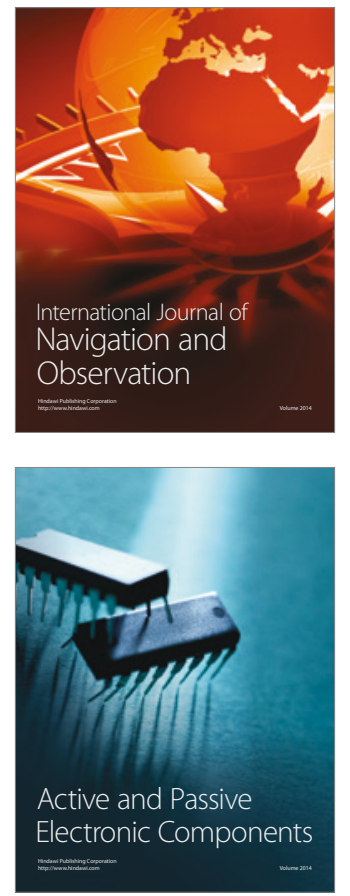
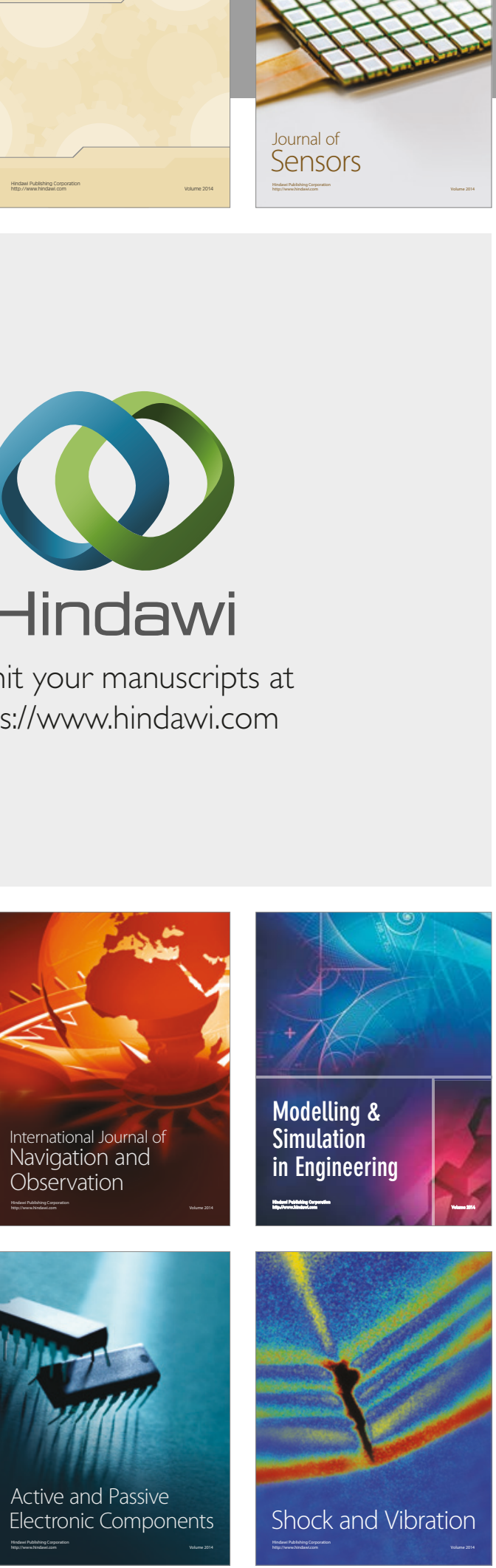
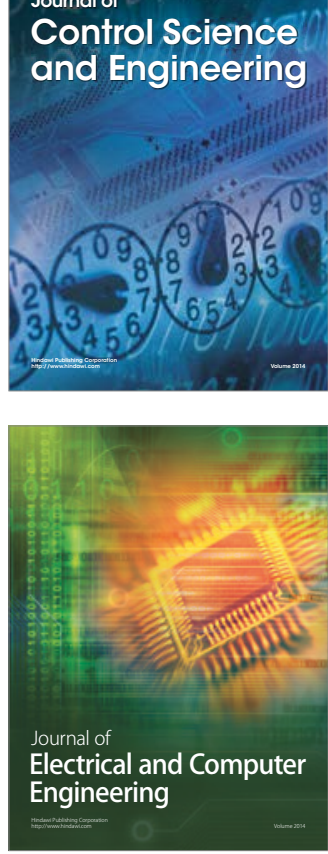

Distributed

Journal of

Control Science

and Engineering
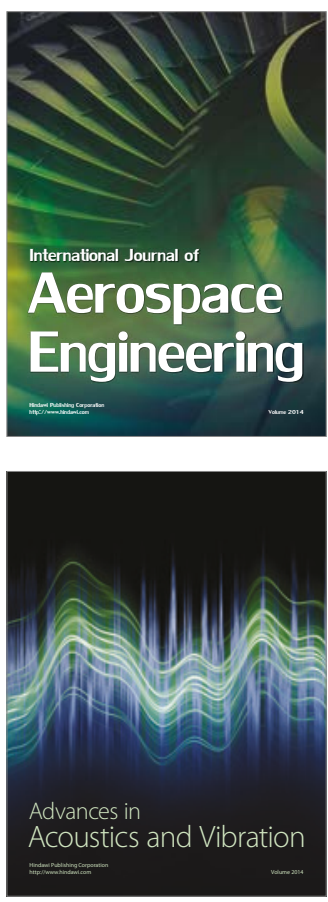

Sensor Networks 